\title{
UN MODELO METODOLÓGICO PARA EL ESTUDIO DEL CUERPO EN INVESTIGACIONES BIOGRÁFICAS: LOS MAPAS CORPORALES ${ }^{\mathbf{1}}$
}

A methodological model for studying the body in biographic research: Body Maps

\author{
Jimena Silva* \\ Jaime Barrientos** \\ Ricardo Espinoza-Tapia ***
}

\section{Resumen}

En este artículo teórico se presenta el modelo metodológico de los Mapas Corporales como una estrategia para la producción de conocimientos sobre el cuerpo en investigaciones biográficas. Este modelo aborda la corporeidad como un lugar por el que fluye el trazado de construcciones intersubjetivas que regulan al sujeto, permitiendo textualizar procesos subjetivos que, con las estrategias tradicionales del método biográfico, quedan invisibles, en tanto, lenguajes semiótico-materiales encarnados. A nivel interpretativo se busca la comprensión del orden normativo inscrito en el cuerpo donde se articulan aspectos relativos a procesos psicosexuales y modelos de género anclados en la cultura, como así también, la expresión de transgresiones o disidencias a las ideologías de género vigentes.

Palabras clave: Investigación biográfica, corporeidad, mapas corporales, género.

\section{Abstract}

This theoretical paper refers to a methodological model of Body Maps as a strategy to create knowledge on corporeity in bibliographic research. This model approaches the body as a place through which the intersubjective construction path regulating the subject flows, allowing the textualization of subjective processes which, together with the traditional strategies of the bibliographic method, remain invisible as embodied semiotic-material languages. At an interpretational level, the paper deals with understanding the normative order of the body where aspects relative to psychosexual processes and gender models rooted in the culture articulate, along with the expression of transgression to current gender ideologies.

Key words: Biographic research, Corporeity, Body Maps, Gender.

\section{LA INVESTIGACIÓN BIOGRÁFICA Y EL ESTUDIO DEL CUERPO}

El interés por el uso de los métodos de la investigación biográfica en las ciencias sociales no es reciente (Bourdieu, 1992; Bengoa, 1999). Más bien, el método biográfico ha tenido un desarrollo bajo distintas perspectivas

\footnotetext{
${ }^{1}$ Agradecimientos: Este trabajo ha sido efectuado con el apoyo del Proyecto Fondecyt 1110301.
} 
epistemológicas e intencionalidades teóricas, llegando a la actualidad con un vigor creciente y énfasis desde enfoques disciplinares diversos.

Lo anterior nos lleva a plantear que, cuando los investigadores sociales asumimos la difícil labor de rescatar historias de vida, dicha misión es posible en la medida que cada sujeto esté dispuesto a trabajar con recuerdos, activando su memoria y organizando su testimonio, sea este oral o escrito.

En la reconstrucción biográfica juega un rol importante la interacción que se logra entre quién narra y el sujeto que interpreta y selecciona los diversos pasajes relatados por el sujeto con quien se efectúa esta reconstrucción. En estas interacciones se produce un juego de intersubjetividades complejo entre los actores del proceso en las que se anudan y desanudan espacios de evocación, dependiendo de los lugares de la memoria que se van iluminando. En el caso de las autobiografias, el sujeto que escribe es el protagonista de los hechos, eligiendo de manera autónoma sus recuerdos y asumiendo diversos roles: narrador, escritor e intérprete.

Sin embargo, la riqueza que poseen los métodos en las investigaciones biográficas entra en tensión cuando incorporamos al cuerpo como un elemento más de evocación de la memoria. En ese sentido, Pujol, Montenegro y Balasch (2003) postulan que la corporeidad en la investigación social ha sido negada, deslegitimando el rol del cuerpo, al ser este vinculado a la carnalidad. Así, la implantación de lógicas de pensamiento binarias han transformado a la dicotomía mente-cuerpo en una forma de estructuración del mundo del sujeto occidental, privilegiando la valoración de lo cognoscente como un valor vinculado a lo social y relegando así a lo corpóreo al ámbito material de la naturaleza.

Dicho de otro modo, el ocultamiento del cuerpo en la investigación social ha sido transversal a las formas modernas de discurso científico en las ciencias sociales, formas herederas del "cogito cartesiano", productor de un Yo descorporeizado y deslocalizado que ha centrado su atención en el poder de la racionalidad.

Por ende, la corporeidad como un lugar de textualización abre nuevas vetas metodológicas para estudiar los procesos biográficos, interpelando a los métodos ya existentes, ya que los modelos biográficos no lograrían recoger en toda su profundidad la experiencia registrada por la memoria del cuerpo, en tanto que un entramado semiótico-material (Haraway, 1995). De modo que autores como Denzin y Lincoln (2005), Pujol et al. (2003) y, Becker (1998), van a señalar que para alcanzar esta forma de memoria corporal es necesario articular el método biográfico con disciplinas y formas de representación de la realidad como el arte plástico, el cine y la danza (Radley, 1990; Cohen, 1999). Lo anterior, sin lugar a dudas, pertenece a un debate relacionado con la caracterización de los distintos momentos de la investigación cualitativa en el mundo anglosajón (Denzin y Lincoln, XXX) y sus implicancias epistemológicas y metodológicas.

Con los mapas corporales, entonces, se conjugan preocupaciones por el sentido y significación del sí mismo y su corporalidad expresada como 
lenguaje, entramadas en la biografía de cada sujeto. Tomando en cuenta las experiencias biográficas encarnadas, la preocupación por el sentido del cuerpo es una de las más enigmáticas y persistentes interrogantes que atraviesa cada existencia, aunque es la carne la que encara resistencias e incógnitas por donde circula el poder social (Foucault, 2011). Por ende, con el mapa corporal se propone elaborar un anclaje material que represente a lo corpóreo: carne, huesos, sangre y todos sus sistemas amalgamados con referentes simbólicos.

El modelo metodológico de "Los mapas corporales" permitiría reivindicar la agencia del sujeto: este texto es mío, porque este cuerpo es mío, subvertiendo mecanismos de sujeción de la experiencia semiótico-material propios de la práctica científica, como el silenciamiento del sujeto que habla, la codificación en categorías de la subjetividad y la subalternización en el proceso de interpretar la experiencia del otro. De modo que mediante este modelo se abre una vía para que el cuerpo "se obstine en ser", lo que significa, que "el cuerpo es y se sale con la suya a pesar del poder y de los estereotipos de género" (Rivera, 2011:59).

A partir de estos actos reapropiadores del ser corporal, el sujeto y quien investiga acceden a la comprensión de mandatos, gestos, actitudes y símbolos articulados al poder social que subordinan al sujeto y se manifiestan en sus prácticas sociosexuales. Tales prácticas se organizan como normativas a las cuales el sujeto - desde su autonomía - puede oponerse o interpretar según su biografía y las decisiones que vaya tomando (Araujo y Martucelli, 2010).

De allí que este artículo busca contribuir a la investigación social biográfica, proponiendo un modelo metodológico orientado al estudio del cuerpo desde su valor simbólico. Este modelo se enmarca en el ámbito de teorías críticas, desde las cuales se busca recuperar dimensiones psicosociales y contextuales de las personas. En ese sentido y siguiendo a Pujadas (2002), se busca provocar rupturas epistemológicas que conducirían a los científicos sociales a fuentes de conocimiento social que profundicen en lo que las personas y los grupos representan con sus cuerpos.

A continuación se desarrollan los niveles de análisis del modelo y la propuesta procedimental para su aplicación.

\section{LOS MAPAS CORPORALES}

El concepto de Mapas Corporales empezó a difundirse como técnica con algunas aplicaciones como El sistema de las Flores de Bach, desarrollado por Dietmar Krämer y Helmut Wild, el año 2000. Este sistema permite que todos sean expertos y se acerquen al cuerpo desde una perspectiva de salud alternativa a la de la medicina occidental y académica. Con otro enfoque, el Instituto de la Máscara de la Universidad de Buenos Aires dio a conocer el trabajo Mapa Fantasmático Corporal de Mario Buchbinder y Elina Matoso (2011), prologado por David Le Breton. Estos autores proponen un dispositivo de intervención creado, entre otros fines, para el 
diagnóstico institucional denominado Mapa Fantasmático Institucional (MFI) (Stopiello, 2011). Estas formas de aplicación ofrecen esta técnica dentro de la lógica diagnóstica y de intervención que permite elaborar un conjunto de saberes bajo una disposición de asimetría de poderes sobre la salud física y mental del cuerpo a partir de saberes expertos.

A diferencia de lo anterior, con el modelo propuesto de mapas corporales en la investigación biográfica no se busca favorecer un proceso diagnóstico ni de intervención, por el contrario, se estimula la emergencia de significados y discursos encarnados en un cuerpo protagonista de la biografía del sujeto. Sus procedimientos buscan articular saberes en una co-construcción de escritura, relato oral y gráfica autobiográfica con las que se elabora una geografía de la experiencia corporal a partir de relaciones interpersonales con figuras significativas y autoanálisis de experiencias que emergen desde los niveles intrapsíquicos entramados con escenarios socioculturales y afectivos donde ocurrieron los eventos seleccionados. La relación que se produce entre el sujeto que produce el mapa corporal con el investigador es dialógica, de manera que se reconoce en quien elabora el mapa corporal la noción de autoría, destacando la agencia y autonomía del sujeto en la producción de saber y verdad. El proceso antes descrito puede resumirse en el siguiente esquema:

\section{FIGURA 1. MODELO INICIAL PARA LA INVESTIGACIÓN CON MAPAS CORPORALES}

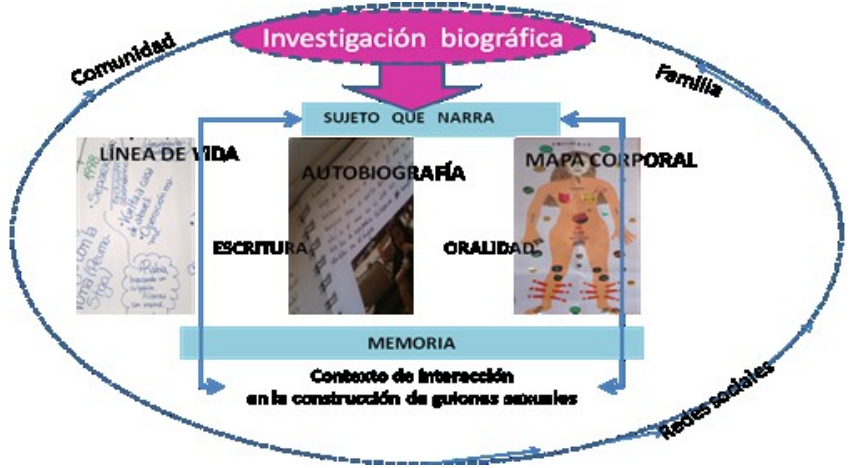

Fuente: elaboración propia.

\section{LOS MAPAS CORPORALES Y SU PROCEDIMIENTO}

\subsection{Organización del grupo}

La selección de participantes para la aplicación de este modelo no presenta restricciones de género, edad, o nivel socioeconómico. Para ejecutar los mapas se requiere que los participantes: a) expresen voluntad y disposición para trabajar en el proceso de reapropiación corporal y autonomía subjetiva a partir de la recuperación de eventos autobiográficos, b) logren continuidad y sistematicidad para participar en todas las sesiones; y c) estén dispuestos a la firma de un consentimiento informado para el uso de autobiografia, mapa corporal y relatos en el proceso de investigación 
biográfica.

2.2. Elaboración de línea de vida

Como herramienta, la línea de vida es un paso primario para organizar en la memoria acontecimientos, como así también, un recurso para encontrarlos fácilmente sí los necesitamos.

La línea de vida se organiza como una gráfica, en la que una persona ubica los nudos de acontecimientos que — para sí — son más relevantes de un periodo de vida, situados éstos de tal manera que muestran conjuntos de procesos biográficos en secuencias de tiempo. Cada nudo representado permite identificar las huellas de eventos biográficos, los cuales dan cuenta de procesos situados en contextos sociopolíticos (económicos, políticos, socioculturales).

\subsection{Escrituras autobiográficas}

La escritura se inicia sobre la base de la producción de líneas de vida. Mediante preguntas reflexivas se estimula a los participantes a abrir los nudos biográficos y se articulan los hechos al contexto donde han ocurrido, identificando personas involucradas y afectos desplegados.

\section{FIGURA 2. EJEMPLO ELABORACIÓN LÍNEA DE VIDA}

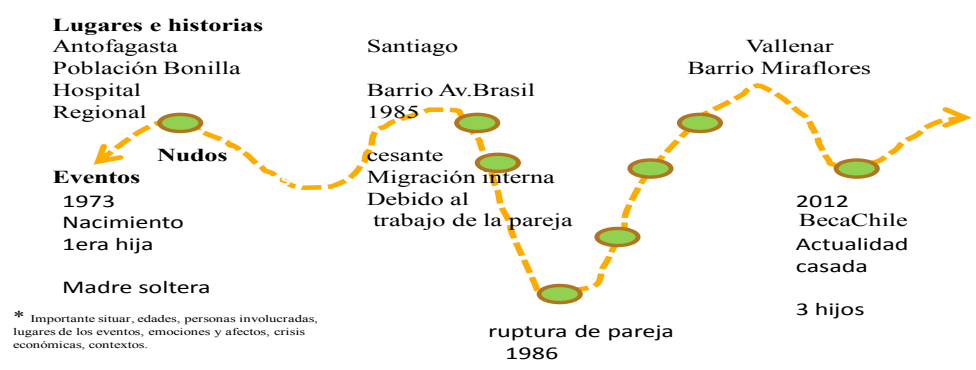

Fuente: elaboración propia

En esta narrativa autobiográfica surgen posibilidades para autointerpretaciones de las relaciones interpersonales con figuras significativas (por ejemplo: sexuales, afectivas, de cuidado, de castigos, entre otras) y análisis de escenarios socioculturales y afectivos donde ocurrieron las experiencias significativas que se han anclado en los procesos asociados a mandatos de género. 
Este proceso de reflexividad estimula la emergencia de emociones desde un enfoque microsocial del contexto social, de relaciones afectivas y de género en la construcción de los protagonistas, facilitando así niveles de comprensión sobre conflictos, elecciones, rupturas, tensiones entre los sujetos y personas significativas de su entorno, entre otras.

Como recomendación, las narrativas que emergen pueden ser grabadas o escritas, de manera que se pueda favorecer la expresión en grupos con dificultades de alfabetización, asimismo, favorecer la reflexividad propia de esta metodología dialógica que vuelve sobre el relato para encontrar nuevas líneas interpretativas de la experiencia a partir del proceso que cada sujeto exprese.

\subsection{Relatar lo escrito}

Cada narración ofrece entradas horizontales, verticales o circulares a experiencias vividas y representadas una y otra vez en la memoria, formando parte de las prácticas humanas (Ferrarotti, 1981; De Villers, 1999; Silva 2008, 2009). Esta parte del procedimiento es de mayor espontaneidad, ya que los fragmentos para narrar son de libre elección. Esta fase implica un esfuerzo por dar sentido al pasado, al presente y a los contenidos asociados al proyecto biográfico o a lo que significa su reformulación o relanzamiento.

Es relevante que durante toda esta fase los participantes desarrollen una postura frente a sus experiencias de dolor o sufrimiento, y así también, a aquellas experiencias satisfactorias. Esta postura promueve su empoderamiento, ya sea desde el autorreconocimiento de sus potencialidades como de la autointerpretación de las opciones de transformación que el sujeto posee.

El rol de los que acompañan esta fase, como monitores o guías, es de escucha activa, facilitando la manifestación de las experiencias vitales, participando con el grupo en las interpretaciones.

\subsection{Mapas corporales}

En esta etapa se recoge el conjunto de los relatos, conversaciones, líneas de vida y autobiografias y estas se disponen para alimentar el mapa corporal. Por tanto, es el momento en el que los nudos biográficos se han abierto a la recuperación, la reflexividad y la textualización. Esta fase se caracteriza por su nivel de densidad simbólica, ya que desde aquí se construye una representación de los sujetos, ofreciendo una interpretación intertextual de la construcción de una biografía corporal.

En este punto del proceso se trabaja bajo criterios de voluntad propia de acuerdo con la aplicación que el investigador, monitor o guía, decida dar al modelo. 
Figura 3. PROPUESTA DE MAPA CORPORAL

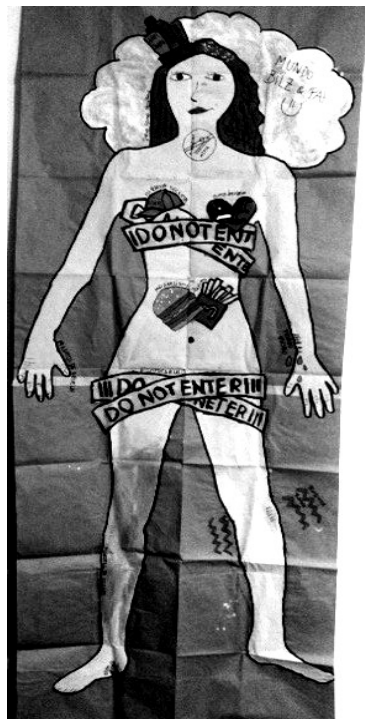

Esta fase se puede iniciar con una consigna del tipo: Dibujaremos símbolos, palabras o mensajes que representen tu cuerpo y las experiencias que tú decidas trabajar.

Esta consigna se debería adaptar al grupo de trabajo. Luego, de este momento, se prepara el papel o tela del tamaño real del cuerpo de la persona y se deja libre la creatividad en cuanto al uso de colores y materiales para las aplicaciones o texturas. A continuación se organiza a los participantes en duplas y se dibuja el contorno del cuerpo en la posición más cómoda para el participante.

Por último, se representan los elementos simbólicos de la experiencia con el cuerpo. Así, por ejemplo, las características de la autoimagen. En otro nivel de representación se registran distintos discursos provenientes de la imagen social construida sobre diversas creencias. Mediante estos discursos se busca facilitar la expresión de inscripciones culturales encarnadas. Finalmente, con el conjunto de elementos simbólicos se trabaja en el proceso de recuperación subjetiva.

Es pertinente explicitar que si bien el modelo suscita interrogantes biográficas y las expresiones de emociones, con este no se busca realizar un trabajo clínico desde el punto de vista diagnóstico.

\subsection{Fase de cierre}

El trabajo detallado de elaboración del mapa corporal se realiza en soledad, en un diálogo con el sí mismo y sus propias autointerpretaciones. Es por esto que, en la etapa de cierre, se genera un espacio donde, tanto, el grupo, 
como, el sujeto deciden voluntariamente compartir su experiencia. Este es un proceso de reflexividad intersubjetivo, donde los participantes han generado oportunidades de reconocerse y hacer un trabajo con aquellos obstáculos que dificultan su bienestar y calidad de vida. Los sujetos dialogan bajo una modalidad grupal sobre sus hallazgos. La metodología permite que discutan e interpelen a sus propios hallazgos graficados en el mapa (ver Figuras 3 y 4).

La dimensión narrativa está compuesta por la recuperación de espacios de memoria y sus interpretaciones, organizados estos espacios en nudos o conglomerados biográficos significativos. Esta dimensión se subdivide a su vez en: a) discursos normativos sobre el cuerpo y restricciones, castigos, mandatos de belleza, estética y de género (Scott, 1990; Muraro, 1994; Butler; 2001; Facio, 1994; 2002; Esteban, 2006; Araujo, 2009): y b) prácticas vinculadas a lo que ocurre a nivel topográfico del cuerpo como el uso de biotecnologías, medicamentos, dispositivos médicos correctivos o estéticos, entre otros.

Con los análisis de estos dos niveles interpretativos - discursos y prácticas - es posible visibilizar discrepancias entre discursos teóricos y las prácticas subjetivas, especialmente respecto de las ideas que dominan sobre los procesos de socialización de género del sujeto. Los mapas corporales permiten así producir conocimientos que revelan que el sujeto es un activo y permanente transformador de las normas sociales, lo que alimenta las argumentaciones en contra de la excesiva linealidad de las concepciones clásicas de los procesos socializadores, los cuales sostienen que se produce "una vinculación más o menos directa entre norma introyectada y comportamiento" (Araujo, 2009:250).

La dimensión gráfica está compuesta por representaciones de autoimagen e imagen social interpretadas por cada sujeto como su "gramática" corporal. Dicha gramática se plasma en formas, colores, tramas e instalaciones, que articulan emociones, sufrimientos físicos, mitos y tabúes inscritos en la carne (Machover, 1949; Barthes, 1986; 1987; Bateson, 1998). Esta dimensión ofrece conocimientos sobre los comportamientos de los sujetos y se fundamenta en el hecho de que los sujetos responden a cierto orden normativo, lo que no garantiza que: "se conduzcan o inscriban sus actos en el marco señalado por este. Hay una brecha entre el ideal y lo procedimental [...] Sin ella, el sujeto, y la necesaria distancia sobre la que se constituye, desaparecería" (Araujo, 2009:254). Interesa lo iconográfico como producto cultural motivado, es decir, construido para producir un efecto, un discurso con intención de expresar los significados de una creación que nos abra puertas interpretativas (Fernández, 2011). 
FIGURA 4. EJEMPLO DE GRILLA DE ANÁLISIS INTERTEXTUAL

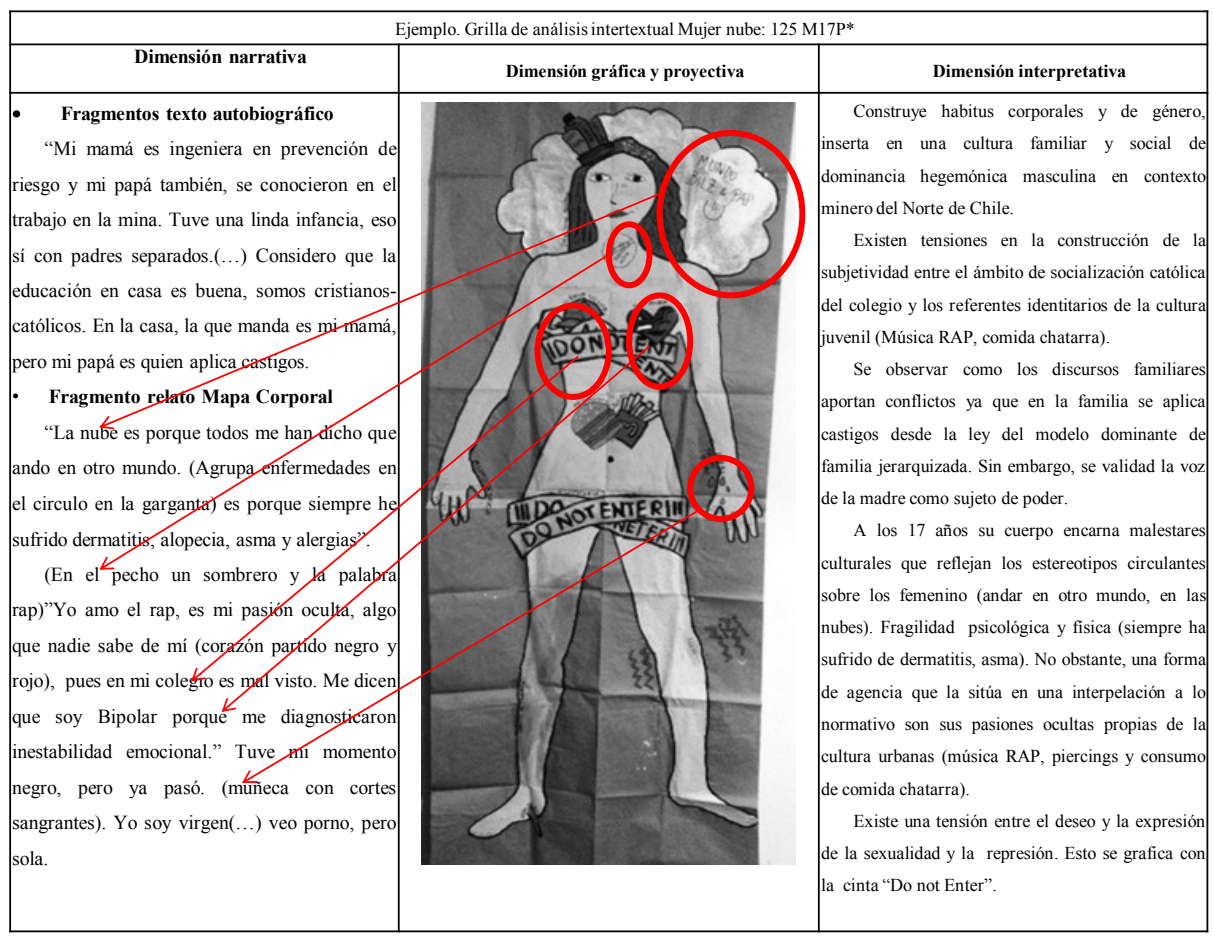

Fuente: elaboración propia

Las posibilidades de las expresiones gráficas (forma, trama, color, texturas, entre otras aplicaciones) al interior de distintos modelos metodológicos son amplias. Por ejemplo, la técnica del dibujo de figura humana (DFH) como método proyectivo desde el campo de la clínica psicológica individual (Kopptiz, 1982; Burns y Kauffman, 1978; Hammer, 1999) utilizado para diagnósticos tanto de adultos como de niños. Desde los estudios culturalistas se reconoce el merito de las investigaciones que recuperan documentos históricos graficados como registros válidos del pasado, y los conciben como construcciones ideológicas, como formas de comunicación humana. La antropología de los medios de comunicación gráfica abarca dos tipos de investigación: primero, estudios de recepción que exploran el impacto de los medios gráficos en una cultura (Caldarola 1990; Dickey 1993); y segundo, el estudio de cómo las personas, generalmente no occidentales, hacen de sus propias producciones (Michaels 1982; Turner 1991).

La dimensión proyectiva está compuesta por un conjunto de productos simbólicos y surge desde la amalgama de las dimensiones narrativa y gráfica. En esta dimensión se mantiene una especial atención al plano inmediato desde donde se elaboran conflictos, los que se plasman en el mapa corporal como textos, diálogos, 
mandatos, reproches y obstáculos que impiden avanzar hacia transformaciones y el bienestar subjetivo, así como rupturas y liberaciones (Silva, 2009).

\section{INTERPRETACIÓN DEL CORPUS DOCUMENTAL: LA DISPOSICIÓN INTERTEXTUAL}

El "análisis" que acompaña el procedimiento se va aplicando progresivamente, en cada etapa de producción de materiales simbólicos. Esta forma de proceder se aproxima a la postura antropológica de campo. El "análisis" se va haciendo con los participantes a lo largo de toda la investigación, y consiste en construir con ellos progresivamente una representación de la cultura encarnada en sus cuerpos.

Para el tratamiento de todos los materiales recolectados en el proceso, se trabaja desde una comprensión intertextual, inspirados en los trabajos de Julia Kristeva (1967), Jaques Derrida (1971), Michel Foucault (1976) y Roland Barthes (1987). Estos autores utilizan la intertextualidad para dar cuenta de las múltiples posibilidades de juegos del lenguaje. Barthes, por ejemplo, habla de un ideal de textualidad donde abundan las redes que actúan entre sí sin que ninguna pueda imponerse a las demás. El texto que se produce usando la intertextualidad es una galaxia de significantes y no una estructura de significados; el texto no tiene principio, pero sí diversas vías de acceso, sin que ninguna de ellas pueda calificarse de principal. Asimismo, los códigos que se movilizan se extienden hasta donde alcance la vista; estos son indeterminables. También, los sistemas de significados pueden imponerse a este texto absolutamente plural, pero su número nunca está limitado, ya que está basado en la infinidad del lenguaje (Kristeva, 1967).

El análisis se produce entrando desde los microtextos seleccionados del conjunto de relatos orales y escrituras autobiografias, articulando con la idea de armar un intertexto con las gráficas de los mapas corporales, sus texturas, color e íconos integrados a las emociones recogidas en el proceso. Se propone no jerarquizar la búsqueda, jugando con los hallazgos como en una red de significados. En este juego, la técnica de interpretación incorpora algunos aportes de la teoría del análisis del discurso (De Villers, 1999; Van Dijk, 2002; Canales, 2000) rompiendo con elementos estructuralistas de su vertiente clásica. Para los dibujos se trabaja con aportes de Bateson (1998), incorporando algunos elementos de las técnicas proyectivas (Machover, 1949) y antropológicas (Barthes, 1987;1986). ${ }^{2}$

Si bien planteamos que esta mirada teórica entrega aportes a la necesidad de

\footnotetext{
${ }^{2}$ Del trabajo de Gregory Bateson se destaca el rol de las interacciones simbólicas y el valor de los significados culturales en las interacciones humanas. Para el caso de Machover, su aporte consiste en realizar una propuesta desde perspectivas psicodinámicas para análisis de elementos proyectivos de los conflictos intrapsíquicos en el uso de metodologías gráficas. Finalmente, en el caso de Roland Barthes, se destacan sus aportes para relevar el valor del color, la imagen, la luz en las creaciones artísticoculturales donde el autor otorga alto valor simbólico al contenido gráfico. Así también contribuye con importantes rupturas metodológicas en la aplicación de la intertextualidad para los análisis de productos culturales.
} 
generar una metodología de trabajo que rescate los lenguajes del cuerpo, sugerimos que la elección teórica a la hora de realizar el acto interpretativo quede bajo la libre elección de quien investiga o aplica el modelo. La idea es que en todo el proceso interpretativo no se pierda la creatividad que este permite, pero por sobre todo el carácter intersubjetivo asociado a la interpretación del material.

Con este modelo aplicado a la investigación social, en su objetivo de producir conocimientos nuevos sobre la corporalidad, se propone trabajar el material para interpretación y análisis en dos momentos: 1) Orden de relatos y materiales 2) organización de temas $y / o$ categorías emergentes en grillas o matrices articuladoras.

A su vez, el primer momento, 1) Orden de relatos y materiales, posee las siguientes etapas:

Etapa (1.A). Proceso de análisis e interpretación: comprensión global del relato autobiográfico, elección de fragmentos o microtextos significativos de acuerdo con los criterios de búsqueda o dimensiones a estudiar.

Etapa (1.B). Organización de fragmentos se organizan en grillas se integran los microtextos del relato que acompañan al mapa corporal intentando armar una red intertextual significante.

Etapa (1.C). Integración de elementos del dibujo del mapa corporal: se arma un nodo compuesto de color, forma de la imagen e íconos, con relatos en una grilla; lo que permite organizar una intertextualidad.

2) Organización de temas y/o categorías emergentes en grillas o matrices articuladoras.

Etapa (2.A). Hallazgos: durante todo el proceso los hallazgos invitan a interpretar y hacer nexos entre un lenguaje y otro, pero cuando se han entramado todos los textos se alcanza un nivel de complejidad interpretativa. Este nivel facilita hacer contrapunto con elementos teóricos coherentes con las búsquedas, que otorgan mayor consistencia y densidad al análisis, para ir respondiendo las interrogantes y los objetivos si se trata de investigación. 


\section{FIGURA 5. EJEMPLO DE INTERPRETACIÓN DEL CORPUS DOCUMENTAL}

\begin{tabular}{|c|c|c|c|c|}
\hline $\begin{array}{l}\text { Fragmento G } \\
\text { Eje: Mujeres } \\
\text { con sus madr } \\
\text { contexto soci }\end{array}$ & $\begin{array}{l}\text { rilla } \mathrm{N}^{\circ} 2 \text { reinte } \\
\text {-Hijas. Sentido } \\
\text { es. Nudos prob } \\
\text { alizador, en la c }\end{array}$ & $\begin{array}{l}\text { erpretación autobic } \\
\text { os y significacione } \\
\text { blemáticos: implic } \\
\text { construcción del s }\end{array}$ & $\begin{array}{l}\text { ografia Camelia: } \\
\text { es que las mujeres-hij } \\
\text { cancias de los discurso } \\
\text { ser sujetos autónomos }\end{array}$ & $\begin{array}{l}\text { las otorgan a las relaciones } \\
\text { ss y prácticas de género del } \\
\text { o restrictivos. }\end{array}$ \\
\hline $\begin{array}{l}\text { Etapa en la } \\
\text { trayectoria } \\
\text { biográfica }\end{array}$ & $\begin{array}{l}\text { Nudos } \\
\text { problemáticos } \\
\text { o } \\
\text { convocantes }\end{array}$ & $\begin{array}{l}\text { Personas } \\
\text { involucradas } \\
\text { Conflictos o } \\
\text { consecuencias } \\
\text { de } \\
\text { interacción la }\end{array}$ & Narración & Interpretación \\
\hline $\begin{array}{l}\text { Adolescencia } \\
13 \text { años } \\
\text { Antofagasta }\end{array}$ & $\begin{array}{l}\text { Valor } \\
\text { simbólico del } \\
\text { cuerpo } \\
\text { articulado con } \\
\text { prácticas y } \\
\text { discursos } \\
\text { sobre la } \\
\text { sexualidad } \\
\text { (educación } \\
\text { sexual, } \\
\text { mandatos, } \\
\text { restricciones, } \\
\text { relaciones de } \\
\text { género } \\
\text { marcadas por } \\
\text { el poder) }\end{array}$ & $\begin{array}{l}\text { Madre, padre, } \\
\text { hija. } \\
\text { y } \begin{array}{l}\text { Experiencias de } \\
\text { violencia y } \\
\text { abuso sexual de } \\
\text { la madre. }\end{array} \\
\text { la }\end{array}$ & $\begin{array}{l}\text { Mi madre tenía } 19 \\
\text { años, (...) cuando la } \\
\text { violaron, yo tenía } \\
\text { como } 13 \text { años } \\
\text { cuando me contó lo } \\
\text { de la violación, sin } \\
\text { muchos retalles, } \\
\text { más que nada para } \\
\text { que me cuidara de } \\
\text { andar sola (...) y me } \\
\text { contó que ella quería } \\
\text { llegar virgen al } \\
\text { matrimonio, no por } \\
\text { moral, (...) más bien } \\
\text { por un r deseo } \\
\text { personal. Después } \\
\text { de la violación ya no } \\
\text { tenía mucho sentido } \\
\text { “cuidarse” así que } \\
\text { comenzó a tener } \\
\text { relaciones con mi } \\
\text { papá, después de } \\
\text { cuatro años de } \\
\text { pololeo. Además } \\
\text { estaba sola en otra } \\
\text { ciudad, tenía más } \\
\text { libertades. }\end{array}$ & $\begin{array}{l}\text { La violación posee un } \\
\text { lugar en la cultura desde } \\
\text { el inicio de las } \\
\text { sociedades. Las mujeres y } \\
\text { los niños/as, cuerpos que } \\
\text { se simbolizan vulnerables } \\
\text { y susceptibles a ser } \\
\text { penetrados: mediante la } \\
\text { seducción, fuerza, culpa, } \\
\text { amenazas provenientes de } \\
\text { otros, en su mayoría } \\
\text { masculinos, que } \\
\text { representan autoridad, } \\
\text { dominio, admiración. }\end{array}$ \\
\hline
\end{tabular}




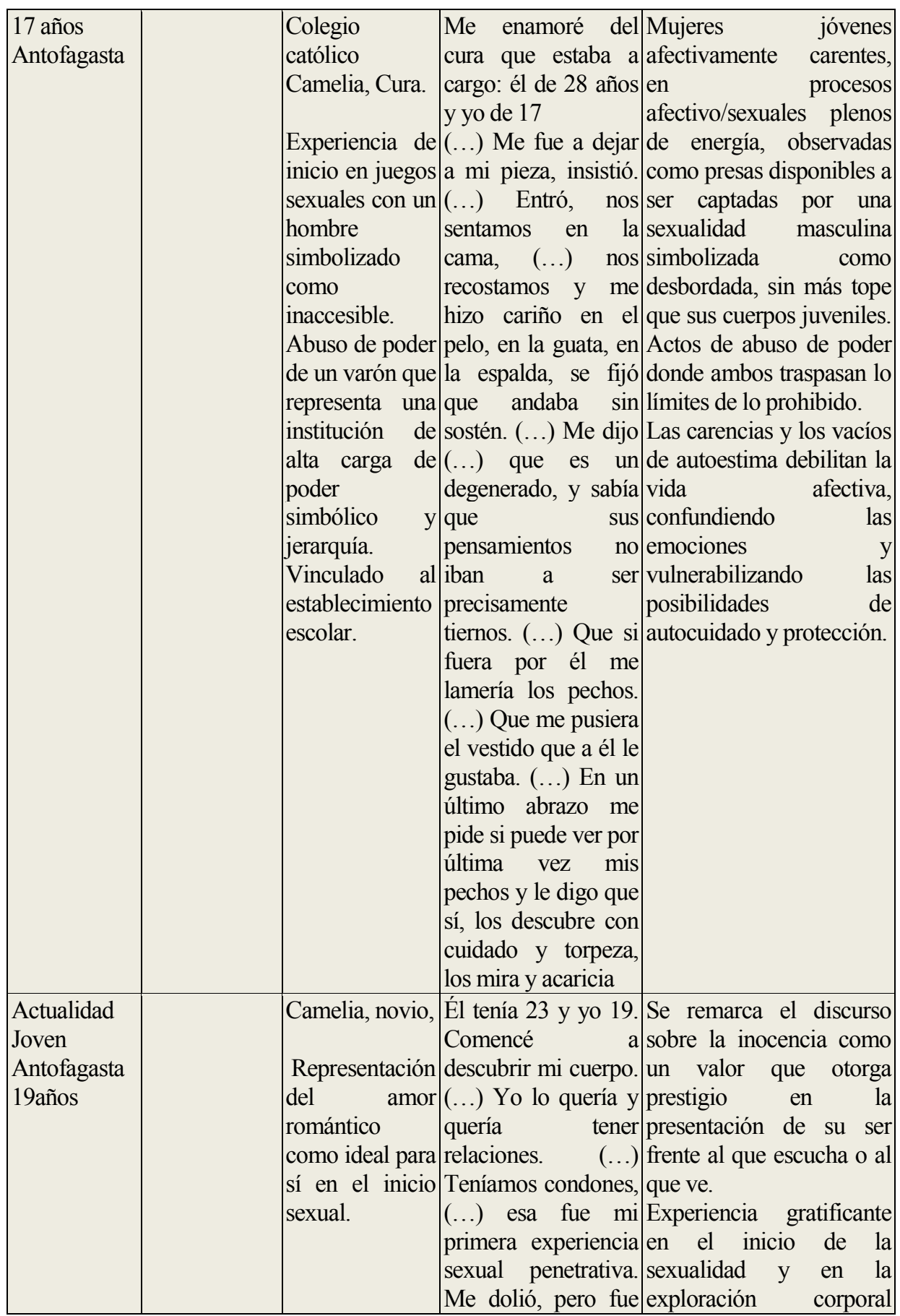




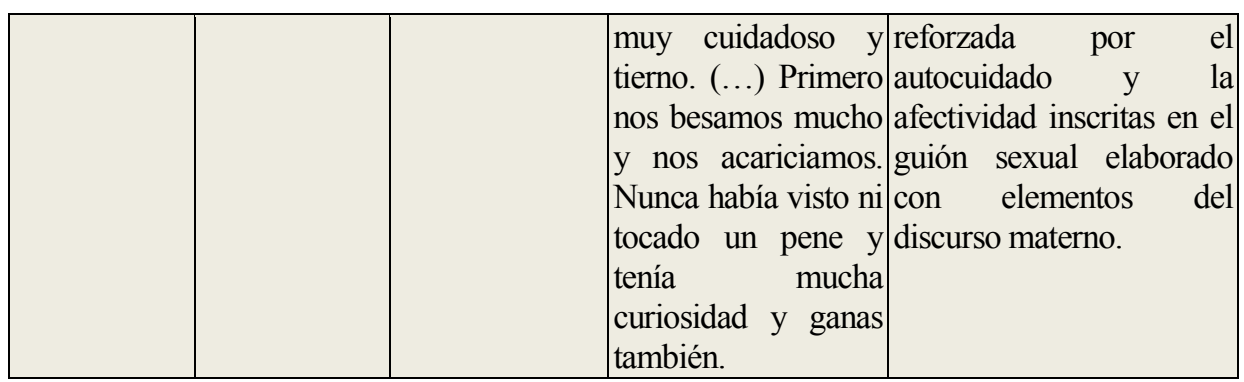

Fuente: elaboración propia

Etapa (2.B) Elaboración de las conclusiones o reflexiones finales: en esta etapa el trabajo del investigador/a consistirá en resolver las interrogantes iniciales sobre el problema, que a partir de la aplicación de este modelo le ha permitido comprender y generar conocimiento nuevo.

\section{DISCUSIÓN}

Las investigaciones que utilizan el método biográfico en ciencias sociales tradicionalmente han dejado vacíos de conocimiento sobre los discursos que el cuerpo produce. En esta experiencia de construirse sujeto sobre la materialidad del cuerpo se articulan imágenes subjetivas con los procesos identitarios y psicosexuales a lo largo de la vida, ubicando al cuerpo en un lugar protagónico en la biografía personal.

En las historias y relatos de vida, si bien se construyen representaciones sobre las experiencias vividas por el cuerpo, es el sujeto que habla el que elabora el discurso sobre él, dejando en silencio la corporeidad desde su propio lenguaje. Por lo tanto, el modelo metodológico de mapas corporales para la investigación biográfica propone recuperar lenguajes corporeizados en sus dominios sexual, cultural, social y de género, abriendo pasajes hacia la compleja relación entre autoimagen, prescripciones culturales normativas y los contextos sociales.

De esta manera estudiar bajo el modelo metodológico estas formas adoptadas desde el orden simbólico expresado en el cuerpo podría ofrecernos información que facilitaría la elaboración de nuevas interpretaciones de ese orden que nos permita generar procesos de reflexividad al interior del método biográfico, a la vez que reconstruirnos como sujetos.

Todas estas articulaciones exigen del investigador avanzar más allá de lo que han alcanzado las herramientas proyectivas en el diagnóstico clínico en Psicología, donde el sujeto es evaluado en una relación asimétrica. Estas articulaciones también permiten avanzar en las investigaciones biográficas con una técnica que contribuye con información relevante sobre experiencias corporales en su complejidad semiótico-material. 
Con los Mapas Corporales se precisa que el protagonista de la biografía se convierta en autor de la interpretación, los cuerpos no son tratados entonces como "objetos" de estudio específicos para su clasificación de sanos o enfermos (lo que llevaría, en cierta forma, a reinstalar el dualismo cartesiano), sino que son reconocidos en amplias dimensiones constitutivas e insoslayables de toda práctica social. Lo anterior cobra sentido en el contexto sociopolítico y cultural latinoamericano, donde las experiencias socioafectivas se construyen en contactos altamente globalizados y fragmentarios de la experiencia del sujeto.

En sociedades como la latinoamericana, con relaciones de género organizadas mayormente en jerarquías de poder, nos construimos, por una parte, de metáforas, ficciones, retazos y suturas y, por otra, somos interpelados a consumir y producir. Estas demandas atrapan al sujeto en una existencia paradójica que muchas veces le enmudece hasta el dolor o la destrucción. En este sentido, este modelo metodológico ofrece estrategias de comprensión del cómo la cultura se hace carne desde discursos socioculturales, entramados en el mundo intrapsíquico, los que se implican en las relaciones interpersonales, dependiendo del tipo de protagonismo que adquiere en cada sujeto, anclado a un conjunto de normativas de la sociedad actual. Así también, abren una opción de renovar diálogos transdisciplinarios entre antropología, sociología, psicología y psicoanálisis. Esto significa que al escuchar los lenguajes del cuerpo, encontramos una vía riquísima de interpretación de conflictos entre pautas normativas ideales de la cultura y la experiencia de trabajo intersubjetivo con las normas inscritas en la carne.

Sin embargo, como todo modelo, este posee limitaciones. Respecto a la organización de los grupos no es recomendado para trabajos con un número elevado de personas, resultando óptimo solo con grupos de entre 15 y 20 personas por taller. Dentro de los procedimientos y sus riesgos, es importante evitar explorar aspectos que trasgredan su intimidad y que expongan a los participantes a los prejuicios y juicios de los demás. Al respecto, por ejemplo, es necesario evaluar previamente la conveniencia o no de realizar espacios mixtos (hombres y mujeres, niños y adultos).

En relación con monitores o guías, requiere que estos posean capacitación en la técnica y sus fundamentos teóricos para su desarrollo. En el proceso pueden contribuir a identificar y a gestionar recursos personales, familiares, comunitarios e institucionales, a los que las personas pueden acudir. Es importante tomar nota atenta de las demandas de las personas, de sus necesidades y requerimientos de apoyo y tramitarlas en las instancias pertinentes. En particular, es preciso atender las demandas de apoyo psicológico, y se debe contar con espacios que permitan prestar una atención especializada.

Una consideración relevante está referida al contexto en que se aplica el modelo, ya que en experiencias en ambientes multiculturales andinos se observaron particularidades por las diferentes interpretaciones y valoraciones simbólicas del cuerpo y de las relaciones sociales. Por ejemplo, en el caso de cosmovisiones andinas, 
el cuerpo poseerá significados diversos de los occidentalizados (Van Kessel, 2008), como es el caso de las cuatro fases del "ciclo vital andino" (Bascopé, 2001). ${ }^{3}$

Estas particularidades culturales, en las diferentes aplicaciones, deberá ser un elemento central en la organización de las estrategias, una recomendación que contribuye al desarrollo del modelo consistirá finalmente en trabajar enfatizando la oralidad y la fase gráfica, introducidas con otras metodologías adecuadas a los contextos socioculturales del grupo.

Universidad Católica del Norte*

Facultad de Humanidades

Escuela de Psicología

Avda. Angamos 0610, Antofagasta (Chile)

jsilva@ucn.cl

Universidad Católica del Norte**

Facultad de Humanidades

Escuela de Psicología

Avda. Angamos 0610, Antofagasta (Chile)

jbarrien@ucn.cl

Universidad Católica del Norte***

Facultad de Humanidades

Escuela de Psicología

Avda. Angamos 0610, Antofagasta (Chile)

respinoza@ucn.cl

\footnotetext{
${ }^{3}$ De acuerdo con Bascopé (2001) y Van Kessel (2008), en el ciclo vital andino el cuerpo refleja la cosmovisión del mundo en cuatro dimensiones; arriba; abajo; izquierda; derecha. El espacio del nacimiento se ubica en la parte "izquierda", que simboliza la relación con los orígenes de la vida. Referido al inicio de la totalidad de los vivientes. El crecimiento está ubicado en la parte de "abajo" en relación con la dimensión de la conservación, restauración y recreación de todo lo creado: la Pachamama representa lo masculino y femenino y sentido de la fecundidad que da la existencia. La muerte se ubica en la parte "derecha", articulado al sentido de la conclusión, cumplimiento, llegada, culminación. Espacio de la proyección, después de concluir una etapa de la vida. Estas representaciones inscritas o encarnadas desde la comunidad lingüística posee un peso fundamental en la socialización corporal de los sujetos. Si bien posee algunos procesos en común con la cultura occidental eurocentrada, los cultos y representaciones corporales andinos, generan interpretaciones duales, vinculados a deidades vigentes en su imaginario que no se encuentran en la occidental (Silva, 2008).
} 
Un modelo metodológico para el estudio del cuerpo en investigaciones biográficas

\section{OBRAS CITADAS}

Araujo, Kathya. "Configuraciones de sujeto y orientaciones normativas", en Psicoperspectivas Individuo y Sociedad. Vol. VIII, $\mathrm{N}^{\circ}$ 2, JulioDiciembre 2009. Web. Consultado junio 10 de 2011.

$<$ http://www.psicoperspectivas.cl/index.php/psicoperspectivas/articl/vie wFile/60/79>

Araujo, Kathya; Danilo Martuccelli. "La individuación y el trabajo de los individuos", en Educação e Pesquisa, São Paulo. Vol. 36, N especial, 2010: 077-091. Web. Consultado: noviembre 28 de 2011.

$<\mathrm{http}: / /$ www.scielo.br/pdf/ep/v36nspe/v36nspea07.pdf >

Barrientos, Jaime; Silva, Jimena. De la restricción hacia la equidad. Chile: Ediciones Universitarias, Universidad Católica del Norte, 2006.

Barthes, Roland. El susurro del lenguaje. Más allá de la palabra y de la escritura. Barcelona: Paidós, 1987.

------ Lo Obvio y lo obtuso. Imágenes, gestos y voces. Barcelona: Paidós, 1986.

Bascopé, Víctor. "El sentido de la muerte en la cosmovisión andina: El caso de los valles andinos de Cochabamba", en Chungara (Arica). Vol. 33, ํ2, julio de 2001: 271-277. Web. Consultado junio de 2012.

$<$ http://www.ifeanet.org/temvar/rev64133211.pdf >

Bateson, Gregory. Pasos hacia una ecología de la mente. Buenos Aires: LohléLumen, 1998.

Becker, Howard. Tricks of the trade: How to think about your research while you're doing it. Chicago, IL: The University of Chicago Press, 1998.

Bengoa, José. "El testigo. Apuntes de clase de un curso de Historias de Vida", en Proposiciones, marzo 29 de 1999:52-74. Impreso.

Bernárdez, Asunción. "Arte postmoderno, ¿Arte feminista? Cuerpo y representación en la sociedad de la información", en Contar con el cuerpo: Construcciones de la identidad femenina. Fernández, A. \& López M. (Coords.). Madrid: Editorial Fundamentos, 2011.

Bourdieu, Pierre. Por una sociología reflexiva. Barcelona: Herder, 1992.

Buchbinder, Mario; Matoso Eliana. Mapas Fantasmáticos del Cuerpo. Buenos Aires: Letra Viva e Instituto de la Máscara, 2011.

Burns, Robert; Kaufman, Harvard. Los dibujos kinéticos de la familia como técnica psicodiagnóstica. Buenos Aires: Editorial Paidós, 1978.

Butler, Judith. Mecanismos psíquicos del poder. Teorías sobre la sujeción. Madrid: Cátedra, 2001.

Caldarola, Victor. Reception as cultural experience: Visual mass media and reception practices in Outer Indonesia. Unpublished Ph.D. diss, Annenberg School of Communication, USA: University of Pennsylvania, 1990. 
Canales, Manuel. Conversaciones para el entendimiento. Escuela de Sociología, Universidad de Chile: Monte hermoso kulturunea, 2000.

Cohen, Cynthia. "A Patchwork of Our Lives: Oral History Quilts in Intercultural Education", en Electronic Magazine of Intercultural Education. Vol. 1, № 3. 1999. Web. Consultado marzo de 2012.

$<$ http://www.eastern.edu/publications/emme $>$

Denzin, Norman; Lincoln, Yvonna. "The Sage Handbook of Qualitative Research. Third Edition. Thousand Oaks: Sage Publications, Inc. Introduction", en The Discipline and Practice of Qualitative Research. 2005: 1-13. Web. Consultado diciembre de 2012.

$<$ www.catedras.fsoc.uba.ar/.../traduccion\%20Denzin\%20_\%20Lincoln>

Derrida, Jacques. De la Gramatología. Buenos Aires: Siglo Veintiuno Editores, 1971.

De Villers, Guy. "La historia de vida como método clínico", en Revista Proposiciones. Vol. 29, marzo 1999: 103-114. Chile: Sur Ediciones. Impreso.

Dickey, Sara. Cinema and the urban poor in South India. NewYork: Cambridge, UniversityPress, 1993.

Esteban, María Luz. Antropología del cuerpo: género, itinerarios corporales y cambio. Barcelona: Ediciones Bellaterra, 2006.

Facio, Alda. "Engendrando nuestras perspectivas", en Revista Nuevas Miradas. Vol No 2, Año 2. 2002. Web. Consultado octubre de 2010. $<$ http://redalyc.uaemex.mx/src/inicio/ArtPdfRed.jsp?iCve=18320201 >

Facio, Alda. Cuando el género suena, cambios trae. Introducción al análisis de género: categorías analíticas básicas. Managua: Programa Interdisciplinario de Estudios de Género, Universidad Centroamericana, 1994.

Ferrarotti, Franco. Storia e storie di vita. Roma: Laterza. 1981.

Fernández, Antonia. "Cuerpo nutricio: iconografía de los discursos de la lactación", en: Contar con el cuerpo: Construcciones de la identidad femenina. Fernández, A. \& López M. (Coords.). Madrid: Editorial Fundamentos, 2011.

Foucault, Michael. Los anormales. Buenos Aires: Fondo de Cultura Económica, Séptima Edición, 2011.

------ Tecnologías del yo. Y otros textos afines. Barcelona: Paidós, 2000.

------ Vigilar y castigar. México: Siglo XXI, 1976.

Haraway, Donna. "Conocimientos situados: la cuestión científica en el feminismo y la perspectiva parcial", en: Ciencia, Cyborgs y Mujeres. Madrid: Cátedra/Universitat de Valencia, 1998.

Hammer, Emanuel. Test Proyectivos Gráficos. Buenos Aires: Paidós, 1999. Hillman, James. El Mito del Análisis. Tres ensayos de psicología arquetípica, 
Madrid: Editorial Siruela, 2000.

Jung, Carl. Arquetipos e Inconsciente Colectivo. Barcelona: Editorial Paidós, 1991.

Kramer, Dietmar; Wild, Hemult. Los Mapas Corporales de las Flores de Bach. España: Editorial Sirio, 2000.

Kristeva, Julia. "Bakhtine, le mot, le dialogue et le roman", en Critique, No 239, 1967: 440-441. Impreso.

Koppitz, Elizabeth. El dibujo de la figura humana en los niños. Buenos Aires: Editorial Guadalupe, 1982.

Le Bretón, David. Sociología del Cuerpo. Buenos Aires: Editorial Nueva Visión, 2002.

Machover, Karen. Proyección de la personalidad en el dibujo de la figura humana. Habana: Cultural, 1949.

Mauss, Marcel. Manual de etnografia, Buenos Aires: Fondo de Cultura Económica, 2006.

Michaels, Eric. TV tribes. Unpublished Ph.D. diss. University of Texas, Austin. 1982. Web. Consultado mayo 03 de 2012.

$<$ http://nimbus.temple.edu/,jruby/wava/eric/>

Muraro, Luisa. El orden simbólico de la madre. Madrid: Horas y Horas, 1994.

Pujadas, Juan José. El método biográfico: el uso de las historias de vida en ciencias sociales. Cuadernos metodológicos, 5. Madrid: Centro de Investigaciones Sociológicas, 2002.

Pujol, Joan; Montenegro, Marisela; Balasch, Marcel. "Los límites de la metáfora lingüística. Implicaciones de una perspectiva corporeizada para la práctica investigadora e interventora", en Política y sociedad. 2003: 40-57. Web.

Consultado Marzo 2012.

$<$ http://www.erevistas.csic.es/ficha_articulo.php?url=oai:revistas.ucm.es :article/24779\&oai_iden=oai_revista269>

Radley, Alan. "Artefacts, Memory and Sense of the Past", en Middleton, D. y Edwards, D. (eds.), Collective Remembering. Londres: Sage. 1990: 4659. Web. Consultado octubre 2011.

$<$ http://www.lboro.ac.uk/departments/ss/staff/staff_biog/radley.html $>$

Ricoeur, Paul. Hermenéutica y psicoanálisis. Buenos Aires: Ediciones La Aurora, 1984.

Rivera, María Milagros. "El cuerpo, el género, lo queer", en Contar con el cuerpo: Construcciones de la identidad femenina. Fernández, A. \& López M. (Coords.). Madrid: Editorial Fundamentos, 2011.

Sánchez, María del Pilar. "Género y Salud", en Contar con el cuerpo: Construcciones de la identidad femenina. Fernández, A. \& López M. (Coords.). Madrid: Editorial Fundamentos, 2011. 
Scott, Joan. "El género: una categoría útil para el análisis histórico", en Historia y Género: Las mujeres en la Europa Moderna y Contemporánea. Valencia: Ediciones Alfons el Magnanim. Institució Valenciana d'Estudios i Investigació, 1990.

Silva, Jimena; Barrientos, Jaime. "Guiones sexuales de la seducción, el erotismo y los encuentros sexuales en el norte de Chile", en Revista de Estudos Feministas. Universidad Federal de Santa Catarina Vol. 16, $\mathrm{N}^{\circ} 2$, 2, 2008: 540-556.

Silva, Jimena. Pachakuti, “ ¿Y el regreso de lo indio en Bolivia?”, en Revista Tercer Milenio. Universidad Católica del Norte, Chile, V13 N15, 2008:76-90.

Silva, Silva. "Relaciones de poder entre mujeres chilenas de dos generaciones. Alianzas, transgresiones, disidencias, oposiciones entre madres e hijas", en Primer concurso de resúmenes de tesis con enfoque de género. Chile: Departamento de Estudios y Capacitación, Servicio Nacional de la Mujer, 2009.

Stoppiello, Luis Alberto. "El mapa fantasmático institucional", en Revista Campo grupal, $\mathrm{N}^{\circ}$ 86, febrero de 2007. 2011:7-9. Web. Consultado marzo de 2012. <http://www.psicosocial.edu.uy/bahia/86.pdf>

Tubert, Silvia. "Desórdenes del cuerpo. El retorno de lo excluido", en: Contar con el cuerpo: Construcciones de la identidad femenina. Fernández, A. \& López M. (Coords.). Madrid: Editorial Fundamentos, 2011.

Turner, Brian. (1989). El cuerpo y la sociedad: Fondo de Cultura Económica. México DF: Prefacio a la edición española.

Turner, Terence. "The social dynamics of video media in an indigenous society: The cultural meaning and the personal politics of video-making in Kayapo communities", en Visual Anthropology Review 7(2): 1991:68-76. Web. Consultado diciembre de 2012. $<$ http://www.antropologiavisual.cl/imagenes9/imprimr/ruby.pdf $>$

Van Dijk, Teun. (2002) "Conocimiento, elaboración del discurso y educación", en Escribanía, 2002: 5-22. Colombia: Universidad de Manizales.

Van Kessel, J.J.M.M. "Vida y fuerza del ayllu Kallawaya", en Volveré Revista electrónica. Año V, N. 30. Marzo de 2008. Web. Consultado mayo de 2012. <http://www.unap.cl/iecta/revistas/volvere_30/articulo2.html>

Weinstein, Dana; Weinstein, Michael. "Georg Simmel: Sociological flaneur bricoleur", en Theory, Culture \& Society, vol 8, 1991: 151-168. Web. Consultado marzo de 2012.

$<$ http://tcs.sagepub.com/content/8/3/151.full.pdf + html $>$ 